\title{
Avaliação sobre a efetividade da metodologia de ensino e capacitação em Bioeconomia oferecida pela parceria Unicamp e BE-Basic Foundation
}

\author{
B. O. ORTEGA' ${ }^{1}$, F. G. VIANA ${ }^{2}$, T. T. FRANCO ${ }^{3}$ \\ 1 e 3 Universidade Estadual de Campinas, Faculdade de Engenharia Química e Núcleo \\ Interdisciplinar de Planejamento Energético \\ ${ }^{2}$ Universidade Estadual de Campinas, Núcleo Interdisciplinar de Planejamento Energético \\ E-mail para contato: b122022@dac.unicamp.br
}

\begin{abstract}
RESUMO - O objetivo deste trabalho foi desenvolver uma análise qualitativa e quantitativa de cursos e eventos promovidos pelo convênio estabelecido entre a Universidade Estadual de Campinas (Unicamp) e a Fundação BE-Basic, com o intuito de estimular o desenvolvimento de recursos humanos necessários para o progresso de novos projetos e produtos relacionados à bioeconomia. Dentre os resultados obtidos pelo mesmo, foram realizados mais de 5 cursos relacionados à área de Bioeconomia, que atingiram cerca de 90 alunos de pós-graduação em disciplinas presenciais e aproximadamente 8000 de diversas nacionalidades no curso oferecido via internet. Além disso, 2 projetos voltados à área já foram iniciados com parcerias entre pesquisadores brasileiros e holandeses.
\end{abstract}

\section{INTRODUÇÃO}

O convênio entre a Unicamp e a Fundação BE-Basic ${ }^{1}$ (Biotechnology based Ecologically Balanced Sustainable Industrial Consortium), firmado em dezembro de 2012, possui como principal objetivo estreitar as relações entre as instituições e estabelecer parcerias em pesquisa, ensino e extensão por meio de cursos de pós-graduação e projetos de pesquisa.

Dentre os procedimentos adotados para cumprimento do acordo, houve a introdução da metodologia de ensino e aprimoramento dos estudantes brasileiros, principalmente na Unicamp, reconhecida por suas pesquisas dirigidas à bioenergia e biocombustíveis.

Em longo prazo, a parceria estabelecida, voltada à sustentabilidade e à bioeconomia, planejou disseminar a educação e a especialização de estudantes e pesquisadores, além de proporcionar o crescimento de pesquisas regionais e internacionais nas áreas supracitadas e a capacitação de recursos humanos para o desenvolvimento de produtos e processos bioeconômicos.

\footnotetext{
${ }^{1}$ Consórcio formado por institutos de pesquisa, universidades e empresas privadas holandesas, que visam desenvolver soluções industriais baseadas em recursos renováveis biológicos para construir uma sociedade mais sustentável.
} 
Com o objetivo de comprovar o rendimento dos cursos, disciplinas e eventos oferecidos pelo convênio Unicamp - BE-Basic, este trabalho pretendeu analisar quantitativa e qualitativamente o andamento e rendimento da parceria estabelecida, embasado nas avaliações dos alunos, suas participações e progressos relacionados a projetos parceiros estabelecidos após ações do convênio.

\section{METODOLOGIA}

Dentre as iniciativas do convênio, foram produzidas e introduzidas no planejamento escolar da Unicamp disciplinas eletivas sobre assuntos até então pouco estudados no Brasil. Merecem destaque os cursos nos temas Multicomponent Mass Transfer (IQ474) e Beyond Bioethanol (IQ 302A e IQ474B), ambos oferecidos pela pós-graduação da Faculdade de Engenharia Química, Communication \& Policy for a Biobased Economy (HO450), realizado pela pós-graduação do Instituto de Economia, e Metabolomics for Microbial Systems Biology (BI100), promovido pela pós-graduação da Faculdade de Engenharia de Alimentos.

Além disso, outra novidade foi a realização do primeiro Massive Online Mass Course $(\mathrm{MOOC})^{2}$ junto à Unicamp, no tema "Technology for Biobased Products", em que professores da Unicamp deram aulas a respeito da história e produção do bioetanol brasileiro.

Ambas as disciplinas e o MOOC abrangeram temas requeridos para processos bioeconômicos, como o estudo da transferência de massa (necessária à aplicação de processos que envolvam biomassa) e o desenvolvimento de estudos de viabilidade para processos de biocombustíveis voltados principalmente para alunos de pós-graduação da Unicamp, mas que também atraíram alunos ouvintes de instituições de pesquisa próximas e empresas privadas especializadas em biocombustíveis.

Durante a programação das disciplinas oferecidas, houve a preocupação de introduzir-se ferramentas de Engenharia para resolução dos exercícios dados em aula, como MATLAB ${ }^{\circledR} \mathrm{e} o$ Mathcad $^{\circledR}$, que oferecem métodos de cálculos e programação direcionados à Engenharia e que muitas vezes não possuem grande ênfase durante a graduação dos alunos.

Juntamente com as aulas práticas realizadas, os cursos contaram com aulas interativas ministradas por especialistas, muitas vezes internacionais, que promoveram a divulgação de oportunidades na bioeconomia e pesquisas de inovação na área, ampliando o campo de pesquisa e trabalho dos pesquisadores.

Complementando a formação técnica dos alunos participantes, houve grande preocupação com a questão do empreendedorismo, ou seja, incentivo aos estudantes de buscar modelos de negócios para implantação no mercado. Dessa forma, agregou-se a necessidade do Brasil, relacionada a novas iniciativas de pesquisa e desenvolvimento na área de bioeconomia, e a possibilidade de idealização e conclusão de projetos de pesquisadores sem grande apoio financeiro.

\footnotetext{
${ }^{2}$ Cursos online e gratuitos, que apresentam conteúdo interativo em formato de vídeos curtos, os quais buscam atingir o maior número de estudantes pelo mundo.
} 
Para comprovação do desempenho dos professores e conteúdo aplicado bem como futura melhoria, a organização preocupou-se em aplicar questionários de avaliação e por vezes trabalhos de conclusão, a fim de comprovar o aprendizado dos participantes e encontrar as faltas cometidas ou assuntos que mereciam maior ênfase em projetos futuros.

\section{Resultados}

Conforme apresentado na figura 1, a introdução de cursos inovadores conseguiu atrair grande contingente de alunos participantes.

\section{Figura 1 - Fluxograma de desenvolvimento do curso MOOC Technology for Biobased Products}

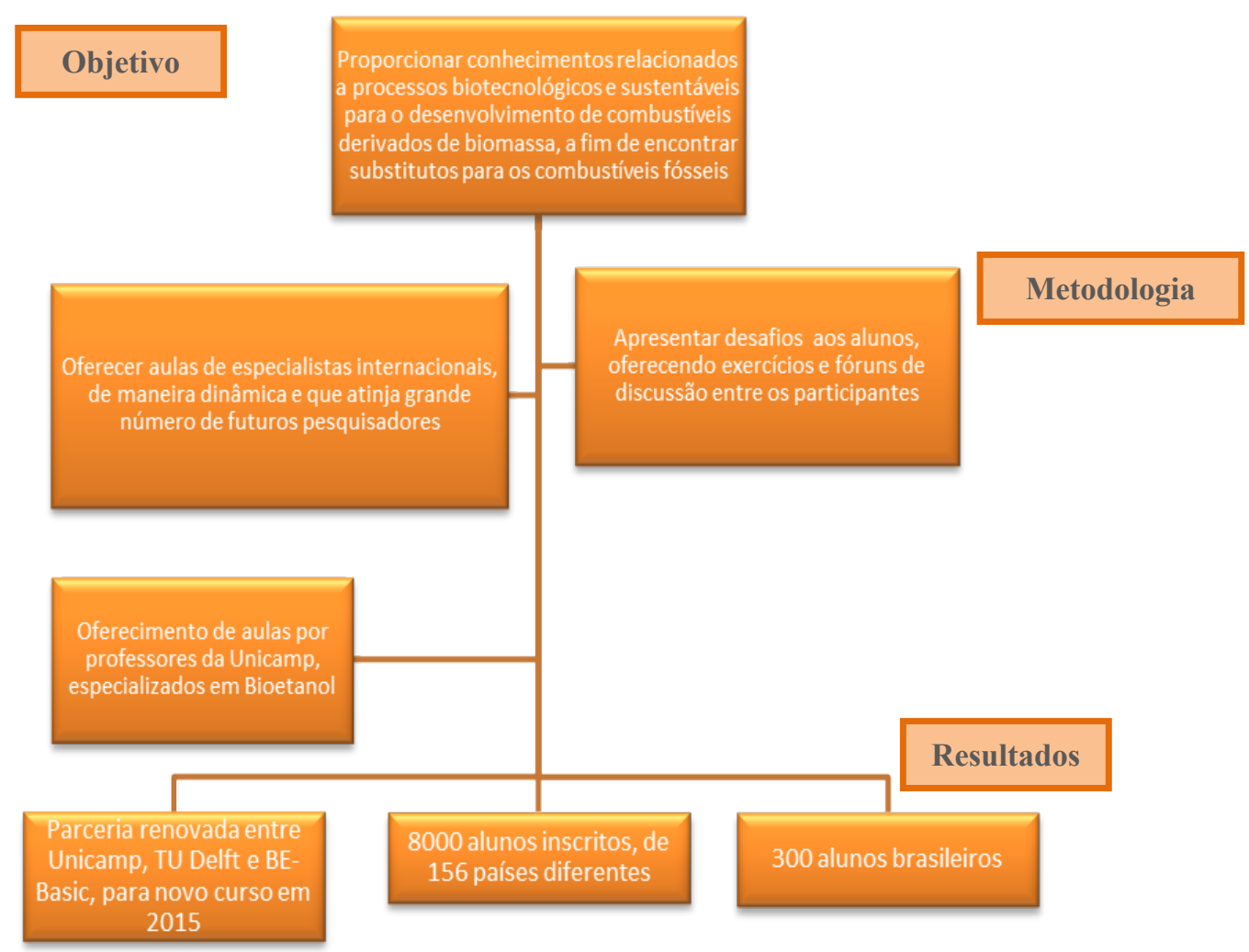

Como exemplos, a introdução do business plan como principal forma de avaliação nas disciplinas Beyond Bioethanol (Figura 2) e Communication \& Policy for a Biobased Economy. Da mesma forma, a realização de um concurso Design Competition dentro do BBEST 2014 - Brazilian Bioenergy Science and Technology Conference ${ }^{3}$ com o objetivo de

\footnotetext{
${ }^{3}$ Conferência Internacional trianual, organizada pela Fundação de Amparo à Pesquisa do Estado de São Paulo (FAPESP) e instituições parceiras, que contou com a presença de pesquisadores, centros de pesquisa e indústrias do ramo de Bioenergia. A última edição do BBEST foi realizada em outubro de 2014, em Campos do Jordão (SP).
} 


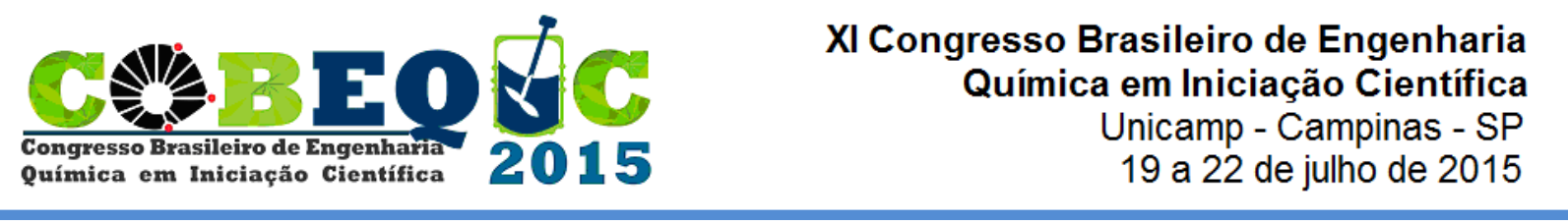

desenvolvimento de um plano de negócios de um processo ou produto economicamente sustentável (Figura 3).

Figura 2 - Fluxograma de desenvolvimento das edições da disciplina Beyond Bioethanol

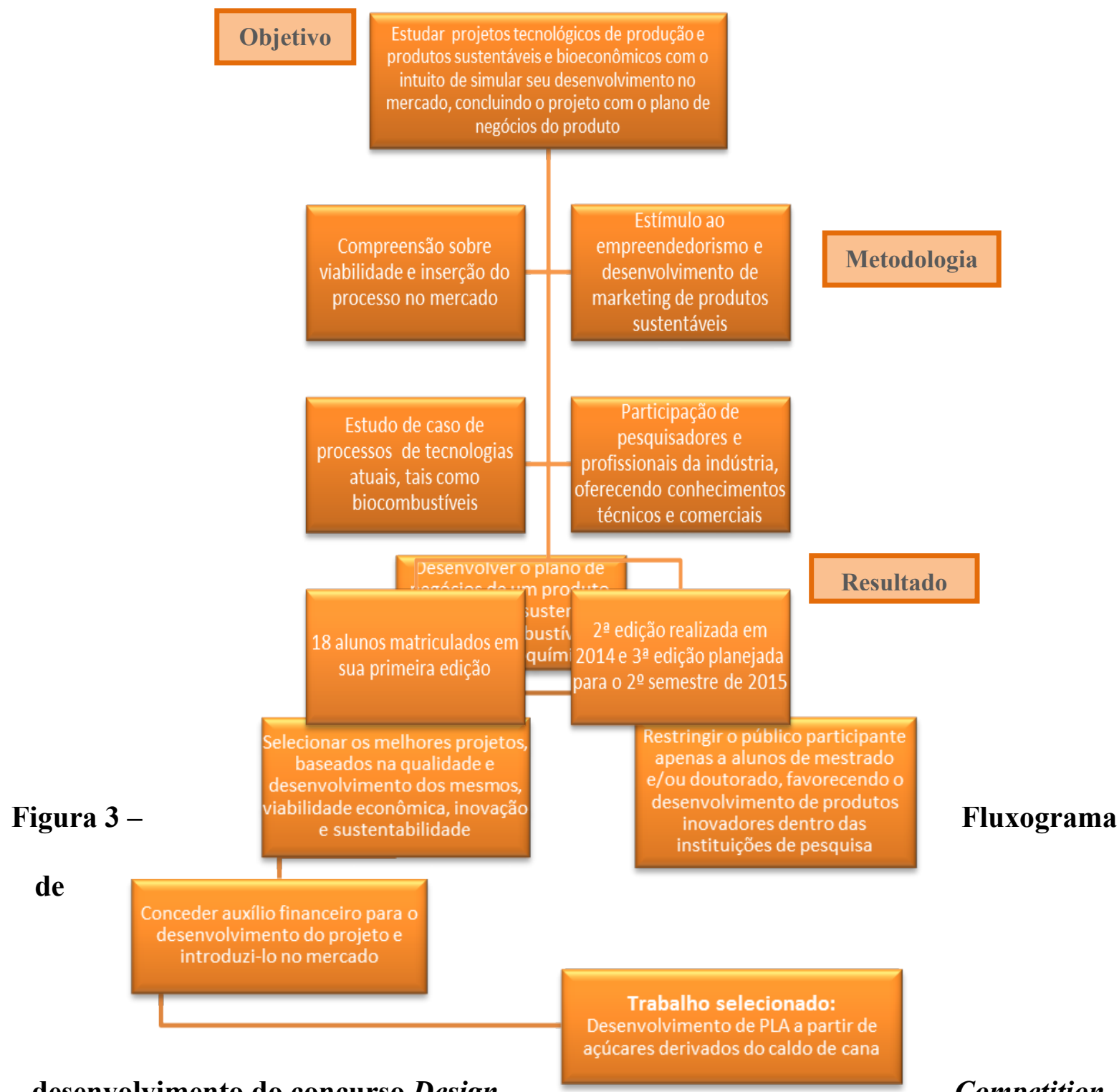

desenvolvimento do concurso Design

Competition

Objetivo

Metodologia 
Por fim, a Fundação BE-Basic, em parceria com professores e pesquisadores da Unicamp, institutos de pesquisa e empresas, realiza eventos especializados de cunho científico em áreas como sustentabilidade, biomassa, resíduos sólidos e sensoriamento remoto. Além de disseminar a temática da bioeconomia, tais eventos objetivam divulgar os atuais projetos apoiados pelo convênio e atrair novas pesquisas com especialistas brasileiros nas áreas relatadas (Figura 4).

Figura 4 - Fluxograma de desenvolvimento do Workshop: Upstream Management: challenges for second generation sustainable production of bioproducts

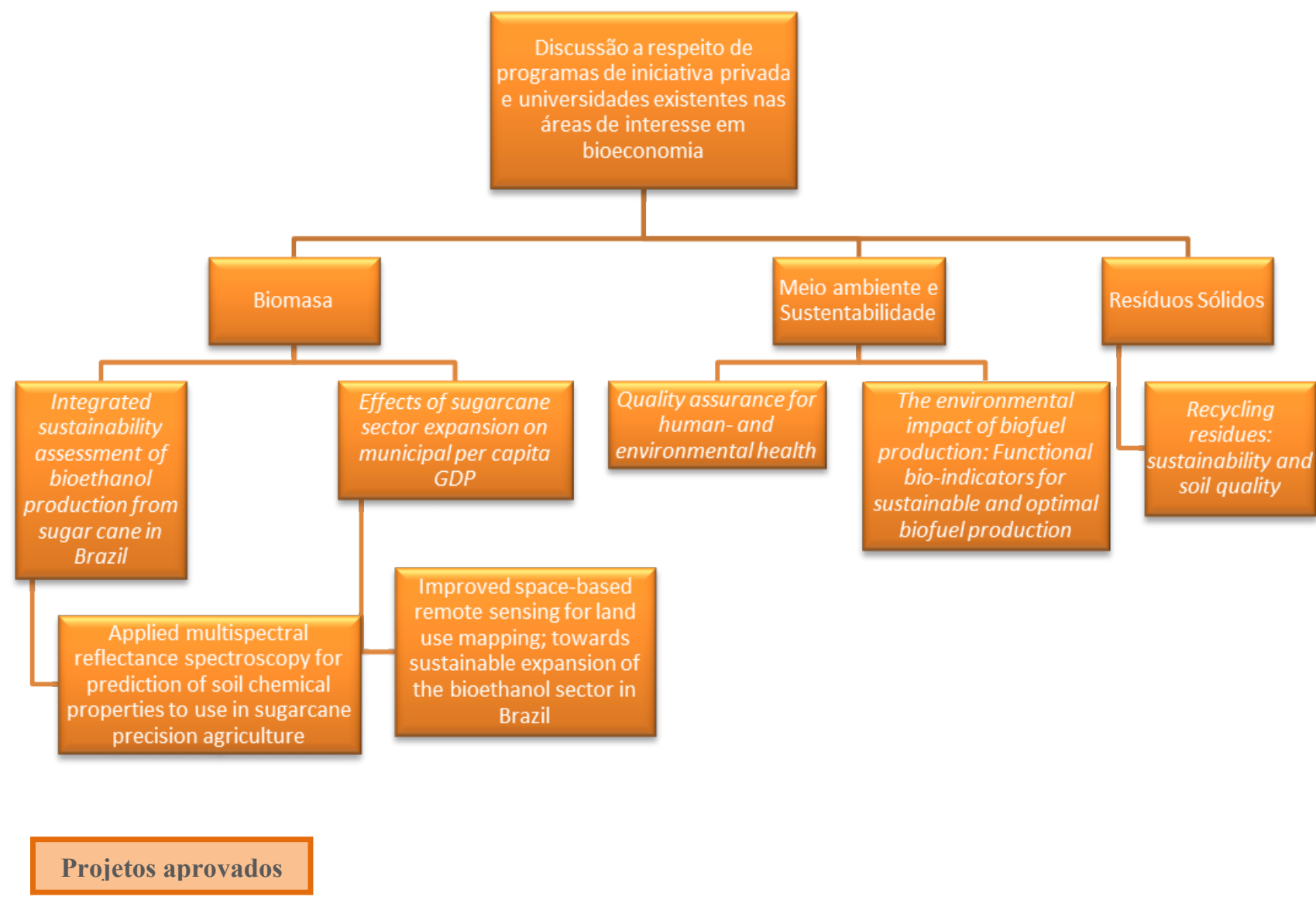


Os fluxogramas supracitados são algumas das mais importantes ações e que obtiveram relevante sucesso em seus objetivos de disseminar a capacitação e aprendizado de pesquisadores e professores e promover aprofundamento da pesquisa voltada à Bioeconomia e sustentabilidade no Brasil.

No entanto, ainda foram realizadas outras atividades que visaram atingir o âmbito de pesquisadores e estudantes da região do estado de São Paulo, como pode ser analisada na tabela 1, em que há a relação do número de atividades e suas respectivas classificações, concluídas até o início de 2014, pelo convênio BE-Basic - Unicamp.

Tabela 1- Resumo de atividades executadas pelo convênio entre 2013 e 2015

\begin{aligned} & \hline Programa de Ensino \\ & BE-Basic Brazil - Unicamp 2013- fev/2015 \\ & \hline Concursos 1 \\ & Disciplinas 5 \\ & Workshops 2 \\ & Cursos à distância 1 \\ & Parcerias 1 \\ & \hline\end{aligned}

\section{CONCLUSÕES}

Como consequência dos trabalhos desempenhados pela Fundação BE-Basic no convênio firmado junto à Unicamp, estão sendo alcançados os resultados esperados, totalizando oito eventos (disciplinas, concursos e workshops) realizados desde 2013, com o retorno positivo dos profissionais envolvidos, oferecendo networking entre pesquisadores brasileiros e internacionais. Além disso, houve a conclusão de trabalhos voltados à bioeconomia e engenharia, centralizados em temas como o processamento de biomassa e biocombustíveis e seus devidos estudos de viabilidade econômica (elaborados na disciplina Beyond Bioethanol).

Em 2015, já foi realizada uma disciplina (Metabolomics for Microbial Systems Biology) e ainda há o planejamento para três workshops, além de uma nova edição da disciplina Beyond Bioethanol, realizada em parceria com a faculdade de Engenharia Química.

Da mesma forma, tais trabalhos proporcionaram a expansão da bioeconomia globalizada e possibilitaram a expansão de parcerias da Fundação com outras instituições brasileiras além da Unicamp, visando atingir outras regiões, como o estado de Minas Gerais. 
O objetivo dessa nova etapa do projeto atrelado ao convênio será proporcionar a capacitação e evolução tecnológica voltada à sustentabilidade e produtos bioeconômicos em outros centros de pesquisa e estudos no país, oferecendo capacitação interativa e prática, com profissionais experts nos temas envolvidos.

\section{REFERÊNCIAS}

BE-Basic Brazil Brochure.

BE-Basic Foundation, Biobased education in The Netherlands Inventory of present and required courses of modules. Disponível em: $<$ http://www.bebasic.org/fileadmin/user_upload/Documenten/PDF/BiobasedEducationNL-Final.pdf $>$ Acesso em 16/03/2015.

LAMPARELLI, R. A. C. Improved space-based remote sensing for land use mapping: towards a sustainable expansion of the bioethanol sector in Brazil. 2013.

MAGALHÃES, P. S. G. Applied multispectral reflectance spectroscopy for prediction of soil chemical properties to use in sugarcane precision agriculture. 2013.

Portal BE-Basic Foundation - BE-Basic Brazil. Disponível em: $<$ http://www.be-basic.org/bebasic-brazil.html >. Acesso em 10/03/2015.

Portal da Unicamp - Unicamp e Delft fazem balanço sobre curso à distância. Disponível em: $<\underline{\text { http://www.unicamp.br/unicamp/noticias/2015/02/13/unicamp-e-delft-fazem-balanco-sobre- }}$ curso-distancia $>$. Acesso em 16/03/2015. 